\title{
On Properties of Torsional Metric Spaces
}

\author{
Hector Alejandro Trejo-Mandujano', George H. Goedecke² \\ ${ }^{1}$ Universidad Autónoma de Ciudad Juárez, Chihuahua, México \\ ${ }^{2}$ Physics Department, New Mexico State University, Las Cruces, NM, USA \\ Email: htrejo@uacj.mx, ggoedeck@nmsu.edu
}

How to cite this paper: Trejo-Mandujano, H.A. and Goedecke, G.H. (2018) On Properties of Torsional Metric Spaces. Journal of Modern Physics, 9, 1793-1806. https://doi.org/10.4236/jmp.2018.99113

Received: July 12, 2018

Accepted: August 6, 2018

Published: August 9, 2018

Copyright $\odot 2018$ by authors and Scientific Research Publishing Inc. This work is licensed under the Creative Commons Attribution International License (CC BY 4.0).

http://creativecommons.org/licenses/by/4.0/ (c) (i) Open Access

\begin{abstract}
We apply general tensor calculus to arbitrary nonrelativistic classical Lagrangian systems and derive general relationships between the torsion tensor and other quantities associated with the coordinate configuration space, such as the metric, the Christoffel symbols, the Euler-Lagrange equations, the affine connections, and the curvature tensor. Using Euler angle metric spaces as examples of spaces with nonzero torsion, we calculate these quantities for nonrelativistic rigid rotators of arbitrary structure. For free rotations, we show that the Euler-Lagrange equations agree with the manifestly correct Euler equations.
\end{abstract}

\section{Keywords}

Torsion, Rigid Rotators, Euler Angle Metrics

\section{Introduction}

In 1922, Élie Cartan included torsion [1] in Einstein's general relativity [2] [3]. Work continued on the Einstein-Cartan theory attempts to associate particle spin and/or rotating matter with torsion [4] [5]. Other investigations considered general effects of torsion in space-time [6] and in a gravitational model of the strong interaction [7]. In addition, several authors (including Einstein) investigated the torsion concept in an attempt to unify electromagnetism with gravity [8] [9] [10]. Trautman [11] also rewrote the Einstein field equations to include nonzero torsion and solved the system for a model consisting of a spinning cloud of dust previous to the big bang. Shapiro [12] investigated effects of including a torsion background in quantized fields. Despite these and many other investigations, the physical role of torsion is still unclear, and modern general relativity is still torsion-free.

In what follows, in Section 2, we apply general tensor calculus to classical 
nonrelativistic Lagrangian systems. We establish general relations between the torsion tensor and other quantities in the coordinate configuration space, such as basis vectors, the metric, the affine connections, the Christoffel symbols, the Euler-Lagrange equations, and the curvature tensor. In Section 3, we consider the often-used "zyz" Euler angle spaces associated with rigid rotators as examples. Very few authors have pursued these examples, e.g. [13] [14]. We outline the development of a symbolic computer algorithm that calculates the essential geometrical quantities for anisotropic rigid rotators. The theory and the computational results, as well as hand calculations, show that the associated Euler angle 3-spaces are indeed non-Euclidean flat spaces with torsion, and that for free rotators the Euler-Lagrange equations agree with the Euler equations derived from conservation of angular momentum. In Section 4, we discuss our results and provide a list of conclusions and suggestions for future work in this area. In Appendix, we report the results of calculations for the popular yaw, pitch, and roll angles.

\section{General Theory}

We represent the $N$ generalized coordinates of a classical system by the set $x=\left[x^{1}, \cdots, x^{N}\right]$, where each $x^{p}$ is an independent real continuous variable that may have any physical dimension and any range. Typical generalized coordinates are Cartesian and radial variables having dimension length, and spherical polar and Euler angles having no dimension. A nonrelativistic system moves on a trajectory in this $N$-dimensional configuration space ( $N$-space) given by

$$
\left[x^{p}=X^{p}(t), p=(1, \cdots, N)\right]
$$

where $t$ is the time. The space may or may not be a Euclidean space $E_{N}$ or a Riemannian space $R_{N}$ [15].

\subsection{Euler-Lagramge Equations}

For nonrelativistic non-dissipative classical systems, the Lagrangian functional $L$ has the form

$$
L=(1 / 2) m g_{p q} \dot{X}^{p} \dot{X}^{q}+m u_{p}(X, t) \dot{X}^{p}-w(X, t) .
$$

Here, the first term is the kinetic energy, $m$ is an overall mass parameter that may be chosen at will, $g_{p q}=g_{q p}$ is called the metric of the $N$-space, and an overdot indicates a time derivative. We follow the extended Einstein summation convention that repeated indices are summed over from 1 to $N$. The $u_{p}(X, t)$ are the covariant components of an $N$-vector potential, and $w(X, t)$ is an $N$-scalar potential energy. We insert the mass parameter so that the distance $\mathrm{d} s$ between infinitesimally separated points is given by the ususal expression, $\mathrm{d} s^{2}=g_{p q}(x) \mathrm{d} x^{p} \mathrm{~d} x^{q}$. If the system contains particles having different masses and/or different moments of inertia, those quantities appear in the metric, ratioed to $m$. The conjugate momenta are 


$$
P_{r}=\partial L / \partial \dot{X}^{r}=m g_{r q} \dot{X}^{q}+m u_{r} .
$$

The Euler-Lagrange (EL) equation is

$$
\mathrm{d} P_{r} / \mathrm{d} t=\partial L / \partial X^{r} .
$$

This equation is assumed to be the correct physical motion equation. When applied to Equations (2) and (1) it yields the detailed EL motion equation

$$
g_{r q} \ddot{X}^{q}+[r, p q] \dot{X}^{p} \dot{X}^{q}=-\partial_{t} u_{r}-m^{-1} \partial_{r} w+\left(\partial_{r} u_{p}-\partial_{p} u_{r}\right) \dot{X}^{p},
$$

where $\partial_{r}=\partial / \partial X^{r}$. The quantities $[r, p q]=[r, q p]$ are the Christoffel symbols of the first kind, defined by

$$
[r, p q]=(1 / 2)\left(\partial_{p} g_{r q}+\partial_{q} g_{r p}-\partial_{r} g_{p q}\right) .
$$

Equation (4) reduces to the geodesic equation if the potentials $u_{p}$ and $w$ are constants.

\subsection{Basis Vectors}

We use the coordinate basis vector approach to general tensor calculus in metric spaces; see e.g. [16] [17]. In this work, we need only the covariant (subscripted) basis vectors $\boldsymbol{e}_{p}(x)$, which are tangents to the coordinate curves [18]. In general, they are not unit vectors and they carry physical dimensions; for example, the covariant basis vectors in an Euler angle 3-space have the dimension of length, as shown below. The generalized dot or inner products among these basis vectors are defined so that

$$
\boldsymbol{e}_{p} \cdot \boldsymbol{e}_{q}=g_{p q} \cdot
$$

We also need the inverse matrix $g^{p q}$ such that $g^{p q} g_{q r}=\delta_{r}^{p}$, the Kronecker delta.

These generalized-coordinate basis vectors at any point $x$ may be expressed as a linear combinations (LC) of a set of $N$ Cartesian unit basis vectors $\hat{\boldsymbol{e}}_{i}, i=(1, \cdots, N)$ that are independent of position $x$ in the $N$-space:

$$
\boldsymbol{e}_{p}(x)=\Lambda_{p i}(x) \hat{\boldsymbol{e}}_{i},
$$

where the Cartesian basis vectors satisfy $\hat{\boldsymbol{e}}_{i} \cdot \hat{\boldsymbol{e}}_{j}=\delta_{i j}$, the Kronecker delta metric. In many cases the generalized coordinates $x^{p}$ may be obtained by a global coordinate transformation $x^{p}\left(\tilde{x}^{i}\right) \leftrightarrow \tilde{x}^{i}\left(x^{p}\right)$ from global Cartesian coordinates $\tilde{x}^{i}$, e.g., consider spherical polar coordinates $\left[x^{p}\right]=[r, \theta, \phi]$ in Euclidean 3-space. Then the matrix $(\Lambda)$ is given by $\Lambda_{p i}=\partial \tilde{x}^{i} / \partial x^{p}$. In such cases, the matrix elements satisfy the integrability condition $\partial_{q} \Lambda_{p i}=\partial_{p} \Lambda_{q i}=\partial^{2} \tilde{x}^{i} / \partial x^{p} \partial x^{q}$. In other cases, such as torsional spaces, this condition is not satisfied, and the space is non-Euclidean. Such matrices $(\Lambda)$ may always be found. They provide the easiest route to detailed expressions for the affine connections and the torsion tensor for Euler angle and other isolated spaces.

\subsection{Affine Connections and Torsion}

In this work, we consider only isolated spaces, as defined recently by Goedecke [18]. In such spaces, the coordinate derivatives of the $N$-space basis vectors at 
any point $x$ may be written as LC's of those same basis vectors:

$$
\partial_{p} \boldsymbol{e}_{q}(x)=\Gamma_{p q}^{r}(x) \boldsymbol{e}(x) .
$$

(This relation is not valid in non-isolated spaces such as some subspaces of higher-dimensional ones, e.g., a 2-sphere embedded in Euclidean 3-space [18].) The coefficients $\Gamma_{p q}^{r}$ are exactly the conventional affine connections. In terms of the matrix $\Lambda$ defined by Equation (7), the connections are given by

$$
\Gamma_{p q}^{r}=\left(\partial_{p} \Lambda_{q i}\right)\left(\Lambda_{i r}^{-1}\right) \text {. }
$$

In isolated spaces, the matrix $\Lambda$ is square, and its inverse exists.

These connections themselves do not constitute a mixed tensor of third rank [1]. However, their antisymmetric parts,

$$
T_{p q}^{r}=(1 / 2)\left(\Gamma_{p q}^{r}-\Gamma_{q p}^{r}\right)=-T_{q p}^{r}
$$

do form a tensor of third rank, called the torsion tensor. In terms of the matrix $\Lambda$,

$$
T_{p q}^{r}=(1 / 2)\left(\partial_{p} \Lambda_{q i}-\partial_{q} \Lambda_{p i}\right)\left(\Lambda_{i r}^{-1}\right) .
$$

Evidently, in any Euclidean space, the torsion tensor is zero in all coordinate systems.

A pertinent general relationship exists among the Christoffel symbols, the symmetrized affine connections, and the torsion tensor. To derive it, we apply Equations (6) and (8), and obtain

$$
\boldsymbol{e}_{r} \cdot \partial_{p} \boldsymbol{e}_{q}=g_{r s} \Gamma_{p q}^{s} \equiv \Gamma_{r, p q} ; \quad \boldsymbol{e}_{q} \cdot \partial_{p} \boldsymbol{e}_{r}=\Gamma_{q, p r},
$$

and add these to get

$$
\partial_{p} g_{q r}=\Gamma_{r, p q}+\Gamma_{q, p r} .
$$

Then, using this equation and Equations (5) and (10), we obtain

$$
[r, p q]=\Gamma_{r,(p q)}+T_{q p r}+T_{p q r},
$$

where the notation $\Gamma_{r,(p q)}$ means the symmnetric part, i.e. $\Gamma_{r,(p q)} \equiv(1 / 2)\left(\Gamma_{r, p q}+\Gamma_{r, q p}\right)$.

\subsection{Curvature}

We assume an isolated space, as above, so that Equation (8) holds. We follow Goedecke's method [18] by calculating

$$
\left(\partial_{p} \partial_{q}-\partial_{q} \partial_{p}\right) \boldsymbol{e}_{r}=R_{r p q}^{t} \boldsymbol{e}_{t},
$$

where

$$
R_{r p q}^{t} \equiv \partial_{p} \Gamma_{q r}^{t}-\partial_{q} \Gamma_{p r}^{t}+\Gamma_{q r}^{s} \Gamma_{p s}^{t}-\Gamma_{p r}^{s} \Gamma_{q s}^{t}
$$

is the usual 4 th rank curvature tensor of the space. But since $\left(\partial_{p} \partial_{q}-\partial_{q} \partial_{p}\right)$ (anything $)=0$ provided the second partials exist, which they do almost everywhere, then $R_{r p q}^{t}=0$ for an isolated space, so such a space is by definition flat. For example, isolated Euler angle 3-spaces are indeed flat, despite 
being torsional and thus non-Euclidean, as we verify by direct calculation of Equation (15) in the next section.

Note that in a torsional space, the affine connections that appear in the curvature tensor are not equal to the Christoffel symbols of the second kind. Instead, they satisfy $\Gamma_{p q}^{s}=\Gamma_{(p q)}^{s}+T_{p q}^{s}$, and $\Gamma_{s,(p q)}$ satisfies Equation (13), whereby

$$
\Gamma_{p q}^{s}=\left[\begin{array}{c}
s \\
p q
\end{array}\right]+T_{p q}^{s}-g^{s r}\left(T_{q p r}+T_{p q r}\right)
$$

is a general relation valid in all $N$-spaces. This relation and Equation (13) were derived by Schouten [15], using quite different nomenclature and notation.

\section{Rotations of Rigid Bodies}

This familiar topic provides calculable examples of the general relations presented above for torsional spaces. Leonhard Euler (1707-1783) introduced the angles named for him as coordinates to describe all possible rotations of any rigid body about a center point, usually the center of mass. These angles expressed as functions of time connect a Cartesian triad of basis vectors fixed in a rotating rigid body to a non-rotating Cartesian reference triad, both with origins at the body center of mass, which itself may be translating nonuniformly under the influence of various force fields. In this section we investigate the properties of Euler angle metric spaces associated with a rigid body having arbitrary structure.

\subsection{Euler Angles and Angular Velocities}

We choose the Euler angles $\left(\alpha^{1}, \alpha^{2}, \alpha^{3}\right)$ corresponding to the $(\alpha, \beta, \gamma)$ used by Arfken [19]. This choice is called the "zyz" set [20], corresponding first to a rotation by $\alpha^{1}$ about the non-rotating Cartesian system z-axis, then a rotation by $\alpha^{2}$ about the new $y$-axis, then a rotation by $\alpha^{3}$ about the final $z$-axis. Altogether there are 12 sets of Euler angles that may be used, but the principal results of our investigation will be the same for all sets. (In Appendix, we present these results for another popular set of Euler angles.)

Let the $\hat{\overline{\boldsymbol{e}}}_{i}$ be the unit basis vectors of the "barred" Cartesian coordinate system that rotates with the object, and the $\hat{\boldsymbol{e}}_{i}$ be the same for the non-rotating ("unbarred") system, where $i=1,2,3$. These basis vectors are related by

$$
\hat{\overline{\boldsymbol{e}}}_{i}=R_{i j}(\alpha(t)) \hat{\boldsymbol{e}}_{j}
$$

where

$$
R(\alpha)=R^{z}\left(\alpha^{3}\right) R^{y}\left(\alpha^{2}\right) R^{z}\left(\alpha^{1}\right)
$$

is the rotation matrix for given set $\left[\alpha^{b}\right]$. The constituent matrices $R^{z}$ and $R^{y}$ are given by

$$
R^{z}(\mu)=\left(\begin{array}{ccc}
\cos \mu & \sin \mu & 0 \\
-\sin \mu & \cos \mu & 0 \\
0 & 0 & 1
\end{array}\right) ; \quad R^{y}(\mu)=\left(\begin{array}{ccc}
\cos \mu & 0 & -\sin \mu \\
0 & 1 & 0 \\
\sin \mu & 0 & \cos \mu
\end{array}\right) .
$$

We may obtain the angular velocity 3 -vector $\omega$ from the defining equation 


$$
\frac{\mathrm{d} \hat{\overline{\boldsymbol{e}}}_{i}(t)}{\mathrm{d} t} \equiv \boldsymbol{\omega} \times \hat{\overline{\boldsymbol{e}}}_{i}(t)
$$

With $\boldsymbol{\omega}=\hat{\boldsymbol{e}}_{i} \omega_{i}=\hat{\overline{\boldsymbol{e}}}_{i} \bar{\omega}_{i}$, we also obtain expressions for the nonrotating and rotating Cartesian components of the angular velocity from Equations (20) and (19):

$$
\omega_{i}=a_{i b} \dot{\alpha}^{b} ; \quad \bar{\omega}_{i}=b_{i b} \dot{\alpha}^{b} .
$$

These equations imply that

$$
b_{i b}=R_{i k} a_{k b} \rightarrow(b)=(R)(a),
$$

where $(b)$ and $(a)$ are $3 \times 3$ matrices given by

$$
\left.(a)=\left(\begin{array}{ccc}
0 & -\sin \alpha^{1} & \sin \alpha^{2} \cos \alpha^{1} \\
0 & \cos \alpha^{1} & \sin \alpha^{2} \sin \alpha^{1} \\
1 & 0 & \cos \alpha^{2}
\end{array}\right) ; \quad b\right)=\left(\begin{array}{ccc}
-\sin \alpha^{2} \cos \alpha^{3} & \sin \alpha^{3} & 0 \\
\sin \alpha^{2} \sin \alpha^{3} & \cos \alpha^{3} & 0 \\
\cos \alpha^{2} & 0 & 1
\end{array}\right)
$$

The relations in this subsection are independent of the structure of the rigid body that rotates with the barred system.

\subsection{Kinetic Energy and Metric}

The rotational kinetic energy for a general rotating rigid body is

$$
T=\frac{1}{2}\left[\bar{I}_{1} \bar{\omega}_{1}^{2}+\bar{I}_{2} \bar{\omega}_{2}^{2}+\bar{I}_{3} \bar{\omega}_{3}^{2}\right]=\frac{m}{2} g_{b c} \dot{\alpha}^{b} \dot{\alpha}^{c},
$$

where $\bar{I}_{i}$ are the three principal moments of inertia in the rotating (barred) body-fixed system; we have chosen a principal axis rotating Cartesian system, as we may always do. Combining Equations (21) and (24) yields

$$
g_{b c}=m^{-1} \bar{I}_{i} b_{i b} b_{i c}=B_{i b} B_{i c},
$$

where for convenince we have defined the quantities

$$
B_{i b}=l_{i} b_{i b},
$$

with principal axis radii of gyration $l_{i}$ given by

$$
l_{i}=\sqrt{\frac{\bar{I}_{i}}{m}} .
$$

Applying Equation (23) and Equations (25)-(27) yields the following metric components:

$$
\begin{aligned}
& g_{11}=l_{3}^{2} \cos ^{2} \alpha^{2}+l_{1}^{2} \cos ^{2} \alpha^{3} \sin ^{2} \alpha^{2}+l_{2}^{2} \sin ^{2} \alpha^{3} \sin ^{2} \alpha^{2} \\
& g_{12}=\left(l_{2}^{2}-l_{1}^{2}\right) \cos \alpha^{3} \sin \alpha^{2} \sin \alpha^{3}=g_{21} \\
& g_{13}=l_{3}^{2} \cos \alpha^{2}=g_{31} \\
& g_{22}=l_{2}^{2} \cos ^{2} \alpha^{3}+l_{1}^{2} \sin ^{2} \alpha^{3} \\
& g_{23}=0=g_{32} \\
& g_{33}=l_{3}^{2}
\end{aligned}
$$

The basis vectors $\boldsymbol{e}_{b}$ of the Euler angle 3 -space yield the correct metric, Equation (25): 


$$
\boldsymbol{e}_{b}=B_{i b} \hat{\overline{\boldsymbol{e}_{i}}} \leftrightarrow \hat{\overline{\boldsymbol{e}}}_{i}=B_{b i}^{-1} \boldsymbol{e}_{b}
$$

\subsection{Affine Connections}

Using Equations (29) and (17), we obtain

$$
\boldsymbol{e}_{b}=\Lambda_{b j} \hat{\boldsymbol{e}}_{j},
$$

where

$$
\Lambda_{b j}=B_{i b} R_{i j} .
$$

Then, using Equation (30) and its inverse yields $\partial_{c} \boldsymbol{e}_{b}=\left(\partial_{c} \Lambda_{b j}\right)\left(\Lambda_{j d}^{-1}\right) \boldsymbol{e}_{d}$, from which we infer an expression for the affine connection

$$
\Gamma_{c b}^{d}=\left(\partial_{c} \Lambda_{b j}\right)\left(\Lambda_{j d}^{-1}\right) .
$$

Equations (30) and (32) correspond to the general Equations (7) and (9). While it is possible to hand-calculate detailed expressions for these affine connections and Christoffel symbols in terms of the Euler angles, the algebra is difficult for a general anisotropic rotator. In Section 3.6, we outline our construction of a symbolic computer algorithm that produces the desired expressions.

\subsection{Euler Equations}

The well-known Euler equations [20] for a torque-free rigid rotator follow from the constancy of the body's intrinsic angular momentum 3-vector $\boldsymbol{S}$. A simple derivation that takes advantage of rotating basis vectors begins with the expression

$$
\boldsymbol{S}=\hat{\overline{\boldsymbol{e}}}_{i} \bar{I}_{i} \bar{\omega}_{i} \text {. }
$$

We apply Equation (20) to obtain

$$
\mathrm{d} \boldsymbol{S} / \mathrm{d} t=0=\hat{\overline{\boldsymbol{e}}}_{k} \bar{I}_{k} \dot{\bar{\omega}}_{k}+\boldsymbol{\omega} \times \hat{\overline{\boldsymbol{e}}}_{i} \bar{I}_{i} \bar{\omega}_{i} .
$$

Expanding the cross-product using $\boldsymbol{\omega} \times \hat{\overline{\boldsymbol{e}}}_{i}=\hat{\overline{\boldsymbol{e}}}_{k} \epsilon_{k j i} \bar{\omega}_{j}$ yields the Euler equations for $\bar{\omega}_{k}$ :

$$
\bar{I}_{k} \dot{\bar{\omega}}_{k}+\epsilon_{k j i} \bar{I}_{i} \bar{\omega}_{j} \bar{\omega}_{i}=0
$$

We expect these equations to be equivalent to the geodesic equation applied to an anisotropic free rotator:

$$
g_{a d} \ddot{\alpha}^{d}+[a, b c] \dot{\alpha}^{b} \dot{\alpha}^{c}=0 .
$$

We may investigate the equivalence of Equations (35) and (36) as follows. First, substitute Equations (21) and (27) into Equation (35), then multiply the whole equation by $b_{k a}$ and use Equation (25), to obtain

$$
g_{a d} \ddot{\alpha}^{d}+\tilde{\Gamma}_{a,(b c)} \dot{\alpha}^{b} \dot{\alpha}^{c}=0,
$$

where

$$
\tilde{\Gamma}_{a, b c}=b_{k a}\left[l_{k}^{2} \partial_{c} b_{k b}+l_{i}^{2} \epsilon_{k j i} b_{j c} b_{i b}\right]
$$

are defined as the "Euler affine connections", with symmetrized forms defined by 


$$
\tilde{\Gamma}_{a, b c)}=(1 / 2)\left(\tilde{\Gamma}_{a, b c}+\tilde{\Gamma}_{a, c b}\right) .
$$

These results show that the relation

$$
\tilde{\Gamma}_{a,(b c)}=[a, b c]
$$

must be satisfied in order that the Euler equations for a free rotator are indeed equivalent to the geodesic equations. The hand calculations of the $\tilde{\Gamma}_{a, b c)}$ quantities are also quite difficult for anisotropic rotators. We verified Equation (40) for such rotators using the algebraic computer algorithms described in Section 3.6 below and also using hand calculations.

\subsection{Isotropic Rotators}

We make hand calculations for an isotropic rotator, defined as any rigid body for which $l_{1}=l_{2}=l_{3}=l$. The metric reduces to

$$
g=l^{2}\left(\begin{array}{ccc}
1 & 0 & \cos \alpha^{2} \\
0 & 1 & 0 \\
\cos \alpha^{2} & 0 & 1
\end{array}\right) .
$$

The Christoffel symbols of the first kind are easily calculated:

$$
[1,23]=[3,12]=-[2,13]=-(1 / 2) l^{2} \sin \alpha^{2},
$$

with $[a, b c]=[a, c b]$ and with all others zero.

One fairly efficient way to obtain expressions for the affine connections in this isotropic case is to begin with Equation (30), put $l_{i}=l$, note that $R_{i j} R_{i k}=\delta_{j k}$, and find that

$$
\boldsymbol{e}_{b}=l a_{i b} \hat{\boldsymbol{e}}_{i},
$$

where $a_{i b}$ are the elements of the matrix $(a)$ given by Equation (23). Then, continue by using the derivatives and the inverse of Equation (43), and obtain $\partial_{c} \boldsymbol{e}_{b}=\left(\partial_{c} a_{i b}\right)\left(a_{d i}^{-1}\right) \boldsymbol{e}_{d}$, which reveals that the affine connections are given by

$$
\Gamma_{c b}^{d}=\left(\partial_{c} a_{i b}\right)\left(a_{d i}^{-1}\right) .
$$

If we then apply $\Gamma_{a, c b}=g_{a d} \Gamma_{c b}^{d}$, we obtain

$$
\Gamma_{1,23}=\Gamma_{3,12}=-\Gamma_{2,13}=-l^{2} \sin \alpha^{2},
$$

with all others zero. Therefore, the symmetrized affine connections are simply equal to

$$
\Gamma_{1,(23)}=\Gamma_{3,(12)}=-\Gamma_{2,(13)}=-\left(l^{2} / 2\right) \sin \alpha^{2},
$$

with of course $\Gamma_{1,(32)}=\Gamma_{1,(23)}$, etc., and with all others zero.Then, using $T_{a b c}=(1 / 2)\left(\Gamma_{a, b c}-\Gamma_{a, c b}\right)$, we obtain the covariant torsion tensor,

$$
T_{a b c}=-\left(l^{2} / 2\right) \sin \alpha^{2} \epsilon_{a b c},
$$

where $\epsilon_{a b c}$ is the completely antisymmetric Levi-Civita 3-index symbol. As indicated by Equation (13), Equation (47) verifies that the symmetrized affine connections are equal to the Christoffel symbols for an isotropic nonrelativistic 
rotator.

It remains to be shown that the intrinsic angular momentum is conserved for the isotropic free rotator. The condition for that conservation is Equation (40). From Equation (39) with $l_{i}=l$ one obtains easily

$$
\tilde{\Gamma}_{a,(b c)}=\left(l^{2} / 2\right) b_{k a}\left(\partial_{c} b_{k b}+\partial_{b} b_{k c}\right),
$$

because the terms in $\epsilon_{k j i}$ in Equation (39) cancel out. Then, using Equation (23), it is a simple matter to hand calculate $\tilde{\Gamma}_{a,(b c)}$ and show that indeed Equation (40) is valid.

These results verify that this Euler angle metric space is indeed a space with torsion, whereby it is not a Euclidean space. It must also be a flat space because it is an isolated space. Using the values of the affine connections $\Gamma_{c b}^{d}=g^{d a} \Gamma_{a, c b}$ that can be obtained easily from Equation (45), it is straightforward if a bit laborious to hand-calculate all the components of the curvature tensor $R_{b c d}^{a}$ defined by Equation (15) and show that they are indeed zero.

\subsection{Anisotropic Rotators}

In this section we investigate properties of the "zyz" Euler angle 3-spaces for rigid rotators of arbitrary shape and structure.

\subsubsection{Equations in Matrix Form}

The computer program that we developed for the general algebraic calculations requires the equations to be in matrix form. In matrix notation, Equation (25) for the metric is simply

$$
(g)=(B)^{\mathrm{T}}(B)
$$

where $(B)^{\mathrm{T}}$ is the transpose of $(B)$. In order to calculate the affine connections, we first define the matrix $\left(M_{c}\right)$ by specifying its elements

$$
\left(M_{c}\right)_{b d}=\Gamma_{c b}^{d} \text {. }
$$

Then Equation (32) in matrix form is

$$
\left(M_{c}\right)=\left(\partial_{c}(\Lambda)\right)(\Lambda)^{-1}
$$

where $(\Lambda)$ is the matrix with elements $\Lambda_{b j}$ defined by Equation (31). Equation (50) is very convenient computationally.

\subsubsection{Method and Results}

Our computer algorithm calculates the affine connections, the Euler affine connections, and the torsion tensor in any Euler angle metric space for any rigid rotator, starting from Equation (50). The algorithm also calculates the corresponding curvature tensor, using Equation (15), and the Christoffel symbols, using Equations (28) and (5). We selected MATLAB [21], [22] to perform the symbolic calculations. Equations (52)-(54) below summarize the results of our calculations for the "zyz" Euler angles. The results are written using the following 
abbreviations:

$$
\sin \alpha^{i} \rightarrow s_{i} ; \quad \cos \alpha^{i} \rightarrow c_{i} ; \quad 2 \alpha^{3} \rightarrow 2 a 3
$$

Equations (52) show the nonzero Christoffel symbols $[a, b c]$, which as expected are symmetric under interchange of their last two indices, and were computationally found to be equal to the corresponding symmetrized Euler affine connections $\tilde{\Gamma}_{a,(b c)}$. This result means that the Euler Equation (35) are indeed equivalent to the geodesic equations for a torque-free rotator. Equations (53) present the nonzero symmetrized affine connections $\Gamma_{a,(b c)}$. Comparison with (52) reveals that these symmetrized connections are not equal to the Christoffel symbols. Equations (54) list the covariant torsion tensor $T_{a b c}$. Note that it is not proportional to the Levi-Civita symbol $\epsilon_{a b c}$. Also, note that in the special case of isotropic rotators, for which all $l_{i}=l$, the quantities in all these equations reduce to their values calculated in Subsection 3.5.

$$
\begin{aligned}
& {[1,12]=c_{2} s_{2}\left(l_{1}^{2} c_{3}^{2}-l_{2}^{2} c_{3}^{2}+l_{2}^{2}-l_{3}^{2}\right)=[1,21]} \\
& {[1,13]=-s_{2}^{2} \sin (2 a 3)\left(l_{1}^{2}-l_{2}^{2}\right) / 2=[1,31]} \\
& {[1,22]=-c_{2} c_{3} s_{3}\left(l_{1}^{2}-l_{2}^{2}\right)} \\
& {[1,23]=-s_{2}\left(\cos (2 a 3) l_{1}^{2}-\cos (2 a 3) l_{2}^{2}+l_{3}^{2}\right) / 2=[1,32]} \\
& {[2,11]=-c_{2} s_{2}\left(l_{1}^{2} c_{3}^{2}-l_{2}^{2} c_{3}^{2}+l_{2}^{2}-l_{3}^{2}\right)} \\
& {[2,13]=s_{2}\left(-\cos (2 a 3) l_{1}^{2}+\cos (2 a 3) l_{2}^{2}+l_{3}^{2}\right) / 2=[2,31]} \\
& {[2,23]=\sin (2 a 3)\left(l_{1}^{2}-l_{2}^{2}\right) / 2=[2,32]} \\
& {[3,11]=s_{2}^{2} \sin (2 a 3)\left(l_{1}^{2}-l_{2}^{2}\right) / 2} \\
& {[3,12]=-s_{2}\left(-\cos (2 a 3) l_{1}^{2}+\cos (2 a 3) l_{2}^{2}+l_{3}^{2}\right) / 2=[3,21]} \\
& {[3,22]=-\sin (2 a 3)\left(l_{1}^{2}-l_{2}^{2}\right) / 2} \\
& \Gamma_{1,(12)}=\sin (2 a 2)\left(l_{1}+l_{2}+l_{3}\right)\left(l_{2}-l_{3}+l_{1} c_{3}^{2}-l_{2} c_{3}^{2}\right) / 4=\Gamma_{1,(21)} \\
& \Gamma_{1,(13)}=-s_{2}^{2} \sin (2 a 3)\left(l_{1}-l_{2}\right)\left(l_{1}+l_{2}+l_{3}\right) / 4=\Gamma_{1,(31)} \\
& \Gamma_{1,(22)}=-l_{3} c_{2} c_{3} s_{3}\left(l_{1}-l_{2}\right) \\
& \Gamma_{1,(23)}=-s_{2}\left(-l_{1}^{2} s_{3}^{2}+l_{1}^{2}-l_{1} l_{2}-l_{3} l_{1} s_{3}^{2}+l_{3} l_{1}+l_{2}^{2} s_{3}^{2}+l_{3} l_{2} s_{3}^{2}\right) / 2=\Gamma_{1,(32)} \\
& \Gamma_{2,(11)}=-c_{2} s_{2}\left(l_{1} l_{2}-l_{1} l_{3}+l_{1} l_{3} c_{3}^{2}-l_{2} l_{3} c_{3}^{2}\right) \\
& \Gamma_{2,(12)}=-c_{2} c_{3} s_{3}\left(l_{1}-l_{2}\right)\left(l_{1}+l_{2}-l_{3}\right) / 2=\Gamma_{2,(21)} \\
& \Gamma_{2,(13)}=s_{2}\left(l_{1}^{2} s_{3}^{2}-l_{1} l_{2}+l_{3} l_{1} s_{3}^{2}-l_{2}^{2} s_{3}^{2}+l_{2}^{2}-l_{3} l_{2} s_{3}^{2}+l_{3} l_{2}\right) / 2=\Gamma_{2,(31)} \\
& \Gamma_{2,(23)}=\sin (2 a 3)\left(l_{1}-l_{2}\right)\left(l_{1}+l_{2}+l_{3}\right) / 4=\Gamma_{2,(32)} \\
& \Gamma_{3,(11)}=l_{3} s_{2}^{2} \sin (2 a 3)\left(l_{1}-l_{2}\right) / 2 \\
& \Gamma_{3,(12)}=-l_{3} s_{2}\left(l_{3}-l_{1} \cos (2 a 3)+l_{2} \cos (2 a 3)\right) / 2=\Gamma_{3,(21)} \\
& \Gamma_{3,(22)}=-l_{3} \sin (2 a 3)\left(l_{1}-l_{2}\right) / 2
\end{aligned}
$$




$$
\begin{aligned}
& T_{121}=\frac{c_{2}}{2}\left(l_{1}^{2} c_{3}^{2}-l_{1} l_{2}-l_{1} l_{3} c_{3}^{2}+l_{1} l_{3}-l_{2}^{2} c_{3}^{2}+l_{2}^{2}+l_{2} l_{3} c_{3}^{2}-l_{3}^{2}\right)=-T_{112} \\
& T_{131}=c_{3} s_{3}\left(c_{2}^{2}-1\right)\left(l_{1}-l_{2}\right)\left(l_{1}+l_{2}-l_{3}\right) / 2=-T_{113} \\
& T_{132}=s_{2}\left(l_{1}^{2} s_{3}^{2}-l_{1}^{2}+l_{1} l_{2}-l_{3} l_{1} s_{3}^{2}+l_{3} l_{1}-l_{2}^{2} s_{3}^{2}+l_{3} L_{2} s_{3}^{2}\right) / 2=-T_{123} \\
& T_{221}=-c_{2} c_{3} s_{3}\left(l_{1}-l_{2}\right)\left(l_{1}+l_{2}-l_{3}\right) / 2=-T_{212} \\
& T_{232}=s_{3} c_{3}\left(l_{1}-l_{2}\right)\left(l_{1}+l_{2}-l_{3}\right) / 2=-T_{223} \\
& T_{213}=\frac{s_{2}}{2}\left(-l_{1}^{2} s_{3}^{2}+l_{1} l_{2}+l_{3} l_{1} s_{3}^{2}+l_{2}^{2} s_{3}^{2}-l_{2}^{2}-l_{3} l_{2} s_{3}^{2}+l_{3} l_{2}\right)=-T_{231} \\
& T_{312}=-l_{3} s_{2}\left(l_{1}+l_{2}-l_{3}\right) / 2=-T_{321}
\end{aligned}
$$

In addition, we calculated the Riemann-Christoffel curvature tensor for an anisotropic rotator using Equation (15) and the calculated values of the affine connections. We found that all components are zero, as predicted.

\section{Conclusions and Discussion}

\subsection{Conclusions}

The principal goals of this work were to investigate properties of affinely connected metric spaces with torsion, and to provide applications to the familiar Euler angle spaces that describe rigid body rotations. The results allow the following conclusions:

1) In any affinely connected metric space, the symmetrized affine connections are equal to the corresponding Christoffel symbols if and only if the covariant torsion tensor is completely antisymmetric or zero (see Equation (13));

2) Any Euler angle 3-space is a flat space with torsion. The covariant torsion tensor is completely antisymmetric if and only if the rigid rotator being described is isotropic;

3) For torque-free rotations of all rigid bodies, the Euler-Lagrange equations that follow from the kinetic energy expressed in terms of the Euler angles agree with the Euler equations that follow from constancy of the intrinsic angular momentum vector;

4) Torsion has no direct effect on the coordinate motions of any non-relativistic Lagrangian system, because the torsion tensor does not appear in the EulerLagrange motion Equations (4);

5) Torsion does have an effect on the detailed form of the Riemann-Christoffel curvature tensor in any space, because the affine connections that appear therein are not equal to the corresponding Christoffel symbols if the space is torsional; see Equations (15) and (16).

As emphasized, the first and last of these conclusions apply in any $N$-space, whereby they apply to relativistic systems. Extension of these results to nonrelativistic systems of many rigid bosies is trivial: We simply have three Euler angles and three CM coordinates for each body in the system. Extension to relativistic many-body systems should be pursued, but it may not be simple, and is beyond the scope of this work. 


\subsection{Discussion}

One topic that merits discussion is the significance of torsion. We should consider two types of significance, geometrical and physical.

The principal geometrical significance follows directly from the definition of the affine connection in terms of coordinate basis vectors, Equation (13), which provides the insight that $\partial_{p} \boldsymbol{e}_{q}=\partial_{q} \boldsymbol{e}_{p}$ in any torsion-free space. At first glance it seems rather unlikely that these partial derivatives would be the same for $p \neq q$. However, as shown earlier, they are equal in all curvilinear coordinate systems $x$ in any Euclidean space. A corollary insight is also well-known: An isolated $N$-space with torsion does not admit any global Cartesian or pseudo-Cartesian coordinate systems, and is thus a non-Euclidean space. Another geometrical significance was noted in conclusion v) just above.

The physical significance of torsion is still unknown. Several possible interpretations and applications have been proposed, as discussed in the Introduction; we will not repeat that discussion here. We have shown that nonzero torsion always accompanies rigid body rotations.

\section{Acknowledgements}

Part of this work is based on part of the Ph.D. dissertaion research of H. T-M.

\section{Conflicts of Interest}

The authors declare no conflicts of interest regarding the publication of this paper.

\section{References}

[1] McConnell, A.J. (1957) Applications of Tensor Analysis. Dover, New York.

[2] Cartan, E. (1922) Comptes Rendus de l Académie des Sciences (Paris), 174, 593-595.

[3] Trautman, A. (2006) Encyclopedia of Mathematical Physics, 2, 189-195. https://doi.org/10.1016/B0-12-512666-2/00014-6

[4] Friedrich, W., Von Der Heyde, P., Kerlick, D. and Nester, J. (1976) Reviews of Modern Physics, 48-3, 393-416.

[5] Petti, R.J. (1986) General Relativity and Gravitation, 18, 441-460. https://doi.org/10.1007/BF00770462

[6] Capozziello, S., Lambiase, G. and Stornaiolo, C. (2001) Annalen der Physik, 513, 713-727. https://doi.org/10.1002/1521-3889(200108)10:8<713::AID-ANDP713>3.0.CO;2-2

[7] Fedosin, S.G. (2009) Journal of Vectorial Relativity, 4-1, 1-24.

[8] Horie, K. (1994) Geometric Interpretation of Electromagnetism in a Gravitational Theory with Space-Time Torsion. ArXiv Eprints hep-th/9409018.

[9] Suhendro, I. (2008) Progress in Physics, 1, 31-37.

[10] Popławski, N. (2010) International Journal of Theoretical Physics, 49-7, 1481-1488.

[11] Trautman, A. (1973) Nature Physical Science, 242, 7-8. https://doi.org/10.1038/physci242007a0

[12] Shapiro, I. (2002) Physics Reports, 357-2, 113-213. 
[13] Goedecke, G. (2014) Statistical Approach to Quantum Mechanics II: Nonrelativistic Spin. ArXiv e-Prints 1408.1721.

[14] Fiziev, P. and Kleinert, H. (1995) Motion of a Rigid Body in Body-Fixed Coordinate Systems-For Autoparallel Trajectories in Spaces with Torsion. ArXiv e-Prints, hep-th/9503075.

[15] Schouten, J. (1989) Tensor Analysis for Physicists. 2d Edition, Dover, New York.

[16] Lichnerowicz, A. (1962) Elements of Tensor Calculus. Methuen, London.

[17] Hartle, J. (2003) Gravity: An Introduction to Einstein's General Relativity. Addison-Wesley, Reading.

[18] Goedecke, G. (2011) The European Physical Journal Plus, 126, 32-41. https://doi.org/10.1140/epjp/i2011-11032-x

[19] Arfken, G. (1985) Mathematical Methods for Physicists. 3d Edition, Academic Press, Orlando.

[20] Goldstein, H. (1980) Classical Mechanics. 2nd Edition, Addison-Wesley, Reading.

[21] (2010) Matlab. The MathWorks Inc.

[22] Handelman, D. and Littlefield, B. (2005) Mastering Matlab 7. Pearson/PrenticeHall, Englewood Cliffs. 


\section{Appendix: Other Euler Angles}

In Equations (A1) and (A2) below we present the results of calculations for anisotropic rigid bodies using "xyz" rotations, with Euler angles known as yaw, pitch, and roll angles. The results have the same character as those in Equations (52) and (54), and they reduce to the correct values for isotropic bodies.

$$
\begin{aligned}
& {[1,12]=c_{2} s_{2}\left(l_{1}^{2}+l_{2}^{2} c_{3}^{2}-l_{2}^{2}-l_{3}^{2} c_{3}^{2}\right)=[1,21] } \\
& {[1,13]=c_{2}^{2} \sin (2 a 3)\left(l_{2}^{2}-l_{3}^{2}\right) / 2=[1,31] } \\
& {[1,22]=-c_{2} c_{2} s_{3}\left(l_{2}^{2}-l_{3}^{2}\right) } \\
& {[1,23]=-c_{2}\left(l_{1}^{2}-\cos (2 a 3) l_{2}^{2}+\cos (2 a 3) l_{3}^{2}\right) / 2=[1,32] } \\
& {[2,11]=-c_{2} s_{2}\left(l_{1}^{2}+l_{2}^{2} c_{3}^{2}-l_{2}^{2}-l_{3}^{2} c_{3}^{2}\right) } \\
& {[2,23]=-\sin (2 a 3)\left(l_{2}^{2}-l_{3}^{2}\right) / 2=[2,32] } \\
& {[2,31]=c_{2}\left(l_{1}^{2}+\cos (2 a 3) l_{2}^{2}-\cos (2 a 3) l_{3}^{2}\right) / 2=[2,13] } \\
& {[3,11]=-\left(c_{2}^{2} \sin (2 a 3)\left(l_{2}^{2}-l_{3}^{2}\right)\right) / 2 } \\
& {[3,12]=-c_{2}\left(l_{1}^{2}+\cos (2 a 3) l_{2}^{2}-\cos (2 a 3) l_{3}^{2}\right) / 2=[3,21] } \\
& {[3,22]=\sin (2 a 3)\left(l_{2}^{2}-l_{3}^{2}\right) / 2 } \\
T_{121} & =c_{2} s_{2}\left(l_{1}^{2}-l_{1} l_{2} c_{3}^{2}+l_{1} l_{3} c_{3}^{2}-l_{1} l_{3}+l_{2}^{2} c_{3}^{2}-l_{2}^{2}+l_{2} l_{3}-l_{3}^{2} c_{3}^{2}\right) / 2=-T_{112} \\
T_{131} & =c_{2}^{2} \sin (2 a 3)\left(l_{2}-l_{3}\right)\left(l_{2}-l_{1}+l_{3}\right) / 4=-T_{113} \\
T_{132} & =c_{2}\left(l_{2}^{2} c_{3}^{2}-l_{2}^{2}+l_{2} l_{3}-l_{1} l_{2} c_{3}^{2}+l_{1} l_{2}-l_{3}^{2} c_{3}^{2}+l_{1} l_{3} c_{3}^{2}\right) / 2=-T_{123} \\
T_{221} & =-\left(c_{3} s_{2} s_{3}\left(l_{2}-l_{3}\right)\left(l_{2}-l_{1}+l_{3}\right)\right) / 2=-T_{212} \\
T_{231} & =-c_{2}\left(-l_{2}^{2} c_{3}^{2}+l_{2} l_{3}+l_{1} l_{2} c_{3}^{2}+l_{3}^{2} c_{3}^{2}-l_{3}^{2}-l_{1} l_{3} c_{3}^{2}+l_{1} l_{3}\right) / 2=-T_{213} \\
T_{232} & =-\left(\sin (2 a 3)\left(l_{2}-l_{3}\right)\left(l_{2}-l_{1}+l_{3}\right)\right) / 4=-T_{223} \\
T_{321} & =l_{1} c_{2}\left(l_{2}-l_{1}+l_{3}\right) / 2=-T_{312}
\end{aligned}
$$

